\title{
An approach to long-term sedative-hypnotic use
}

\author{
This article was published in the following Dove Press journal: \\ Nature and Science of Sleep \\ 30 March 2012 \\ Number of times this article has been viewed
}

\section{Azmeh Shahid \\ Sharon A Chung \\ Ron Phillipson \\ Colin M Shapiro}

Department of Psychiatry, University of Toronto and Toronto Western Hospital, University Health Network, Toronto, Canada
Correspondence: Azmeh Shahid Department of Psychiatry, University of Toronto and Toronto Western Hospital, University Health Network, 399 Bathurst Street, Toronto, M5T 2S8, Canada

$\mathrm{Tel}+$ I 4166035273

Fax +I 4I67030507

Email azmehs@hotmail.com
Abstract: Insomnia is a common, often chronic medical disorder with significant medical and socioeconomic repercussions. However, unlike other medical conditions, there is intense debate as to whether the long-term treatment of insomnia is clinically appropriate. The perceived deleterious side effect of sedative-hypnotic medications may result in patients remaining untreated or undertreated. This review proposes that a more subtle approach needs to be taken in the management of patients with chronic insomnia and that long-term use of the newer sedative-hypnotics may be a feasible and effective treatment option when used in conjunction with thorough medical assessment and regular patient follow-up. This review discusses these issues and discusses the pros and cons of long-term sedative-hypnotic use.

Keywords: insomnia, long-term use, hypnotics

\section{Introduction}

\section{Chronic insomnia: a medical condition that requires long- term treatment?}

The treatment of insomnia has been controversial and under debate for many decades. The medical community has been generally against the long-term use of sedativehypnotic medications. This opinion is not solely based upon reasoned interpretation of the evidence.

The National Commission on Sleep Disorders Research ${ }^{1}$ has described chronic insomnia as an epidemic affecting 30 million Americans, and a Canadian consensus statement ${ }^{2}$ has identified chronic insomnia as a significant health problem. Chronic insomnia is linked to substantial psychosocial, occupational, health, and economic repercussions, results in increased overall morbidity and mortality, and constitutes a drain on healthcare resources. ${ }^{3-7}$ There is little doubt that insomnia is a significant health problem with marked socioeconomic impact. ${ }^{8}$ Many family physicians have the task of treating patients over the long-term. ${ }^{9}$ This does not mean that long-term use of sedative-hypnotics is always warranted; it could be a form of convenience to "get rid of a problem." For some physicians there is a marked dichotomy between what could be viewed as an "ivory tower view" - ie, sedative-hypnotics are "bad" and should be limited in use - versus the "real world" experience of widespread long-term use.

According to the National Institute of Mental Health Consensus Conference, sleep-promoting drug therapy should be considered for patients when they are significantly troubled by the presence of inadequate sleep, or when the physician is concerned about the deleterious impact of disturbed or inadequate sleep on the 
patient's health safety and well-being. ${ }^{9} 10$ This statement is misleading as pharmacologic treatment may not be suitable for every patient complaining of long-term insomnia. Treatment strategies require an understanding of the underlying psychological, social, behavioral, and organic factors. ${ }^{11}$ For example, patients may exaggerate the difficulty they have in falling asleep or underestimate the amount of time they are able to sleep. These are patients who complain of insomnia but on the objective measures do not have insomnia. Trajanovic and colleagues ${ }^{12}$ compared patients who overestimated their sleep time to those who underestimated their sleep time. They defined a condition opposite the previously described sleep underestimation, and named it "positive sleep state misperception." The authors proposed a new model that incorporates both ends of the sleep misperception spectrum.

Cognitive behavior therapy for insomnia (CBTi) is a non-pharmacologic management option for patients with chronic insomnia. It has been shown to produce a clinically significant outcome in the treatment of insomnia with sustained improvement in the 6-month period following treatment. The review by Sivertsen and colleagues suggested that CBTi intervention produced better results when compared to treatment with zopiclone for both the short-term and long-term treatment of insomnia in the elderly. ${ }^{13}$ Unfortunately, improvements are generally realized more quickly with pharmacologic treatment as compared with CBTi and this may account for the low compliance and greater dropout rate with $\mathrm{CBTi} .{ }^{14} \mathrm{~A}$ combination of pharmacologic management and CBTi may be a useful strategy to take advantage of the earlier sleep improvements with pharmacotherapy and the sustained long-term benefits of CBTi, but it has been cautioned that this needs to be decided based on individual patient needs. ${ }^{15}$ Other drawbacks of CBTi include the high initial costs of treatment, no insurance coverage for this treatment for some individuals, availability issues related to the lack of trained CBTi therapists, and that CBTi may be less effective in elderly patients. ${ }^{16}$

In the review by Edginger and Krystal ${ }^{17}$ it was suggested that there is a dichotomy between subjective and objective findings and most of the data available are not conclusive. However, there appears to be sufficient evidence to suggest that subjective and objective insomnia sub-types may suffer from distinctive forms of sleep-related pathophysiology. Others have shown the association of hyperarousal and insomnia. Patients with chronic insomnia have physiological activation that results in increased heart rate, hormone metabolism, and body and brain metabolic rate. This can result in a number of medical problems such as hypertension. In a recent review by Riemann, ${ }^{18}$ the concept that the hyperarousal processes play an important role in the pathophysiology of primary insomnia was highlighted. It is therefore preferable to assess the severity of sleep disruption by evaluating daytime functioning, particularly daytime somnolence. ${ }^{16}$ This would not address the concern of the patient who is "wired" during the day but lacks restful and uninterrupted nocturnal sleep.

\section{Insomnia and depression}

Another important medical consideration is the comorbidity between insomnia and depression that is frequently encountered in clinical practice. Sleep problems are consistently reported in relation to major depression, with insomnia as the most common symptom. ${ }^{19-21} \mathrm{~A}$ study in the general population showed that $40 \%$ of subjects with insomnia presented with a mental illness within 6 months versus $16 \%$ of subjects without insomnia. ${ }^{4}$ At 1-year follow-up, patients with insomnia at baseline were observed to have a greater risk of developing major depression compared to those without insomnia. ${ }^{21}$

The overlap in symptoms of insomnia and depression can at times render it difficult to diagnose and treat the patient despite taking a detailed clinical history. When faced with combined insomnia and depression, management of insomnia is often overlooked. In a study of depressed patients with insomnia, eszopiclone and fluoxetine co-therapy, versus fluoxetine alone, significantly reduced insomnia symptoms and improved ratings of depression, suggesting that treatment of insomnia is an important component of effective management of patients. ${ }^{22}$ Along these lines, use of a sedating antidepressant such as mirtazapine has been shown to improve both depressive symptoms and sleep quality. ${ }^{23}$ We have shown benefits of using tryptophan (which has sedative effects) over placebo in relation to mood improvement. ${ }^{24}$ Guidelines on the treatment of depression recommend that antidepressant treatment be continued for at least 6 months in order to achieve a $70 \%$ or better reduction in the risk of relapse. ${ }^{25}$ Despite insomnia being one of the DSM-IV criteria symptoms for the diagnosis of major depressive disorder, and persistent insomnia being an important risk factor for new or recurrent major depression, ${ }^{3,4,23,24}$ the above guidelines suggest only "symptomatic" management of insomnia "early in treatment" of depression. ${ }^{25}$ If long-term use of antidepressants is recommended in order to achieve optimal patient management, one finds it difficult to comprehend why the same case is not made for sedative-hypnotics treatment of patients with chronic insomnia. Therefore, primary insomnia or insomnia as a symptom of depression necessitates aggressive 
management since untreated insomnia is a risk factor for new or recurrent depression.

\section{Overcoming negative perceptions}

If one accepts that long-term sedative-hypnotics may be required for some chronic insomnia patients, then side effects are a consideration. Kramer ${ }^{26,27}$ echoed the expert advice against using long-term sedative-hypnotic treatment "because of concerns about residual sedative effects, memory impairment, falls, respiratory depression, rebound insomnia, medication abuse, dose escalation, dependency and withdrawal difficulties, and an increased risk of death possibly associated with the current sedative-hypnotic medications." However, he noted that

worries about these potential problems are challenged by the widespread clinical practice of using sedative-hypnotic drugs long-term without any of these difficulties developing and with patients who feel their sleep and daily function is improved with the nightly use of their sleeping pill.

One of the major concerns with long-term sedative-hypnotic use has been the impact of benzodiazepines, in particular, on memory. An exhaustive review of this issue is beyond the scope of this paper but studies do not conclusively support a deleterious impact of benzodiazepines on cognitive function. Our study of 289 sleep clinic patients concluded that benzodiazepine use had no detectable effect on subjective memory difficulties during chronic use. ${ }^{28} \mathrm{~A}$ follow-up investigation of benzodiazepine withdrawal looked at $\operatorname{cognition}^{29}$ and found small yet subtle and reversible effects of long-term benzodiazepines use on speed-dependent tasks in older adults. There are similar findings in the literature that report no difference between long-term benzodiazepine users and anxious untreated participants on a battery of cognitive tests. ${ }^{30}$ In contrast, a widely quoted cohort study reported an association of chronic benzodiazepine intake and deficits on tests of visuospatial processing and sustained attention. ${ }^{31}$ However, it should be noted that only one of five recall measures revealed benzodiazepine-associated impairment. It should also be noted that a study in the elderly also reported cognitive deficits with long-term use of benzodiazepine in those without previous cognitive deficits. ${ }^{32}$

Despite ample evidence to the contrary, another widely held belief is that sedative-hypnotics lose their efficacy in the long-term. Evidence that the deleterious effects of sedativehypnotics may be overrated and that longer use is effective comes from Adam et $\mathrm{al}^{33}$ who showed that sleep was longer and less broken on nitrazepam and that no tolerance was obvious after 2 months' use. This paper was notable for its evaluation of growth hormone secretion. The lack of any associated decrease in the blood level of growth hormone suggests that the metabolic functions associated with slow wave sleep may not be impaired by nitrazepam administration. In another study from the same group, the observation was that the benzodiazepines lormetazepam (2 $\mathrm{mg})$ and nitrazepam $(5 \mathrm{mg})$ appeared to improve sleep after 24 weeks of treatment when compared with continuous placebo use. ${ }^{34} \mathrm{It}$ is concluded that benzodiazepines remain effective for at least 24 weeks but that a period of disturbed sleep may be expected after withdrawal. The strength of this latter study is the control group who took placebo for the full 32 weeks, providing good scientific evidence of maintenance of effectiveness. It is also important to emphasize that the side effect profile of newer sedative-hypnotics has a real advantage over that of benzodiazepines. ${ }^{2}$

Earlier randomized trials have assessed continuous use of sedative hypnotics for a period of 3 months or less: zolpidem -5 weeks; ${ }^{35}$ zaleplon -4 weeks; ${ }^{36}$ and temazepam - 12 weeks of use. ${ }^{37}$ A 6-month randomized, controlled trial of nightly eszopiclone treatment, versus placebo, on measures of quality of life, work limitations, fatigue, sleepiness, insomnia severity, and subjective sleep and daytime function observed sustained improvements in all variables in patients with primary insomnia. ${ }^{38}$ Sustained improvement in total sleep time and/or reductions in sleep latency and number of awakenings after 1 year of sedative-hypnotic treatment has been reported in several patient populations: adults with primary insomnia treated with eszopiclone ${ }^{39}$ chronic psychiatric inpatients who took $0.5-1.0 \mathrm{mg}$ of triazolam; ${ }^{40}$ primary care patients receiving $10-20 \mathrm{mg}$ of zolpidem; ${ }^{41}$ sleep clinic patients with chronic insomnia treated with clonazepam or alprazolam; ${ }^{42}$ and, older insomnia patients administered zaleplon. ${ }^{43}$ However, data on whether the improvements in sleep persist with treatment over the course of several years remains lacking.

Other concerns with long-term sedative-hypnotic use are tolerance and rebound insomnia upon discontinuation. In a 6-month trial of eszopiclone in adult insomnia patients across a range of ages, ${ }^{44}$ including the elderly ${ }^{43}$ no evidence of tolerance was observed despite the drug dosage remaining constant throughout the study and no decrement in treatment efficacy. The Ancoli-Israel study ${ }^{43}$ goes on to report that while some rebound insomnia was evident after abrupt termination of treatment, the sleep variables in the washout period remained improved compared to baseline. This latter finding belies one of the previously firmly held convictions that "insomnia patients show a full return to their symptoms after discontinuing sleeping pills since sedative-hypnotics don't treat the underlying cause of the insomnia." ${ }^{25}$ Morin and 
colleagues reported that temazepam is a safe sedativehypnotic for use by older adults over an 8-week treatment period, with few adverse effects and that with time patients are able to put up with these side effects. ${ }^{46}$

$\mathrm{Kramer}^{27}$ notes that the negative perception about the use of benzodiazepine sedative-hypnotics continues to dictate clinical practice and quotes Regestein and $\mathrm{Reich}^{47}$ who provide a list of cautionary "expert opinions" on the use of sedative hypnotics that illustrates the negative view. The experts advise: "(1) that sedative-hypnotic drugs grant no long-term relief; (2) that the risks outweigh the benefits; (3) that they work largely through placebo effects; (4) that they should never be prescribed for more than a few days nor for more than "occasional" use; and (5) that there is no indication for chronic use and such is always contraindicated." A number of the drawbacks that form the basis of the argument against long-term usage of sedativehypnotics are documented in a well-known and quoted review published in 1990 by Gillin and Byerley. ${ }^{48}$ These authors discouraged prolonged use of sedative-hypnotics due to the lack of research data on the long-term use of sedative-hypnotics.

Over twenty years have passed, during which a number of studies have investigated, and have largely refuted or tempered these "drawbacks," especially for the newer benzodiazepine receptor agonists (eg, the Z-drugs: zopiclone, zaleplon, zolpidem). A review of the "Z" drugs ${ }^{49}$ makes the point that their primary difference from the benzodiazepines is their short half-life and limited duration of action, which accounts for their low risk of residual daytime effect especially with regards to daytime psychomotor performance and memory tasks which are virtually unimpaired by treatment with the " $Z$ " drugs. Nonetheless, it remains virtually impossible to overturn the persistent conviction that long-term sedative-hypnotic treatment is to be avoided. ${ }^{44}$

A further problem is that many patients, possibly echoing their family physicians views, are cautious about taking sedative-hypnotics over the long-term. While this may be an understandable reaction, it is similarly not appropriate when a patient remains untreated or undertreated because their physician refuses to recommend medical management with sedative-hypnotics for as long as is required. It is not a valid perspective when considering long-term treatment of other medical disorders (eg, hypertension or diabetes) to rely on personal (patient's or doctor's) opinion rather than evidence-based medicine. This should be the primary factor guiding clinical management of insomnia.

There is the additional concern that there is a reluctance to examine the option of long-term drug treatment of insomnia. Kramer ${ }^{27}$ purported that "the hazard ratio for prescription sleeping pills was significantly higher than for valium and librium." This suggests the possibility of a specific risk factor for some subclasses of sedative-hypnotic compounds. A significantly greater danger has been shown to be more associated with amitriptyline overdose relative to other tricyclics. ${ }^{50}$ This was previously suggested by other studies examining suicide and risk of death due to overdose of antidepressant drugs. ${ }^{51-53}$ This notion of variation within a class of drugs vis-à-vis danger is not unique and even more variability may be expected between classes of sedative-hypnotics. This to some extent would make one think that if there is a substantial difference in the risk associated with taking amitriptyline versus clomipramine (which a-priori we would not have anticipated) then there is a real likelihood of differential risks with different classes of sedative-hypnotic agents. ${ }^{54,55}$

\section{An examination of the pros and cons}

Bliwise ${ }^{56}$ recommended that the adverse effects of sedativehypnotic drug use, especially benzodiazepines, could be minimized by the following:

1. Using as low a dose as possible

2. Using the drug every third or fourth night

3. Advising patients of potential interactions with alcohol and other drugs

4. Weighing relative risks and carry-over effects

5. Finite or time-limited therapy

6. Gradual termination of therapy.

Of these six points we would regard only the middle two as truisms. Some would argue (the authors among them) that point 1 may lead to inadequate treatment. Further, if sleep worsens due to medical or psychosocial reasons, then the insufficient level of medication would increase the patient's tendency to self-upregulate their dose, reinforcing their belief that "more is better" and foster greater drug dependence in the patient. We favor titration of the initial dose to the patient's needs, as is the case with the majority of prescriptions in other areas of medicine, including psychiatry.

With regard to point 2, this could condition patients to resort to sedative-hypnotic drugs whenever they have difficulty sleeping. We have often preferred using sedativehypnotic medication daily as a course of treatment for a period of 4-8 weeks to break the pattern of concern and expectation about whether the individual will or will not sleep. It also allows for an improvement in sleep quality, in the case of the newer sedative-hypnotic drugs, and slow wave sleep has been shown to increase over time with zopiclone 
treatment, ${ }^{57}$ although not all reports agree with this observation. The analogy can be made that this is a way of building up sleep quality much as an exercise program might improve fitness. Although we have previously routinely used 4 weeks of pharmacological treatment, we have unpublished data supporting the notion that for some individuals taking a sedativehypnotic drug, in whom we have recorded the sleep pattern week by week, sleep quality fluctuates over the first 5-6 weeks but levels out with good sleep efficiency and normalized sleep architecture in the 7 th and 8 th weeks of sedative-hypnotic use. Hence, too short a duration of sedative-hypnotic use may be counter-productive by not allowing for recovery from disrupted sleep. We would recommend a "course of treatment" of 8 weeks with a prior explanation indicated in the following paragraph.

We standardly use the analogy of a bone fracture for patients. ${ }^{58}$ In this analogy the "plaster cast" is the sedativehypnotic and the duration of 2 months makes sense for the patient to allow a healing process and return of normal sleep architecture to occur. There is the further caveat that this occurs better with the non-benzodiazepine sedative-hypnotics that are less likely to suppress slow wave sleep and REM sleep and avoid rebound insomnia on drug withdrawal. A second caveat is that this treatment approach works for $70 \%$ of patients, $20 \%$ of patients return to pre-medication insomnia and sleep architectural disruption on cessation of sedative-hypnotics (this is akin to giving the person with a broken leg a walking stick), and $10 \%$ have no benefit. It is the middle group of $20 \%$ who are candidates for long-term sedative-hypnotic management. A key issue in the first part of this strategy is to provide a script for 8 weeks and to plan a review after 12 weeks, ie, 4 weeks after sedative-hypnotic withdrawal.

We consider points 3 and 4 to be motherhood statements. With regards to point 5: finite or time limited therapy; this speaks to our point that chronic insomnia, when other options are exhausted, requires chronic treatment. The notion of other long-term disorders, eg, hypertension or diabetes, being treated only for the short-term or intermittently would be derided but this is accepted for insomnia. This fact is widely recognized by many family physicians but the reluctance of many sleep specialists and psychiatrists to allow patients access to long-term sedative-hypnotics may cause unnecessary suffering. The evidence indicates that many patients will use a constant medication dose for a chronic sleep problem. This is further supported by a study ${ }^{59}$ of 3000 primary care patients with chronic insomnia prescribed long-term sedative-hypnotics on an as-need basis. Despite chronic use, the patients were capable of limiting their medication intake and, rather than increasing their dosage, actually tended to decrease the use of sedative-hypnotics over time. This is also in keeping with viewing insomnia as a comorbid condition and not merely a symptom of another condition which may require longer treatment.

It should be kept in mind that the two major categories of treatment, non-pharmacological and pharmacological, are not mutually exclusive and that the best management may involve combining both, with non-pharmacological treatment given concurrently with the sedative-hypnotic medication and continued for a longer period. ${ }^{60}$ The efficacy of this strategy for insomnia management has been well demonstrated. ${ }^{61-63}$

Turning to the last point that medication needs to be withdrawn gradually, we agree with this statement as it applies to high dose benzodiazepines. However, with the newer agents (most notably, the " $Z$ " sedative-hypnotics) this requires reconsideration. Our greatest experience would be with zopiclone. In a paper we published some time ago, ${ }^{64}$ we evaluated 154 patients (mean age of 50 years) referred for insomnia and dependent on benzodiazepine medications. The study showed that an abrupt switch to a non-benzodiazepine medication, in this case zopiclone, was best if the preceding benzodiazepine dose was not high. Further, all patients in this study coped well after an abrupt cessation of zopiclone use. Our interpretation is that after a return of normal sleep architecture, abrupt discontinuation of a standard dose of one of the newer sedative-hypnotics is not problematic. The requirement of "gradual withdrawal" may in part reflect the REM rebound associated with benzodiazepine withdrawal.

A follow-up interview of 99 out of the 134 patients in the above study, 14 to 21 months after withdrawal from 1 or 2 months of zopiclone treatment, found that the majority (81 of 99) had remained off sedative-hypnotic medication. These results were better than any in the literature at that time and showed that after long-term benzodiazepine use with a switch to zopiclone for 1 month and with subsequent abrupt zopiclone withdrawal one can maintain a lasting improvement and a drug free state. ${ }^{65}$ Similarly, Lemoine and Ohayon ${ }^{66}$ noted that discontinuation of benzodiazepines is possible in over three quarters of cases, provided that the prescribing physician adheres to a precise withdrawal protocol. Patients on zopiclone were less likely to use sedative-hypnotics during the week of full withdrawal and were more satisfied with sleep than when treated with benzodiazepines. ${ }^{65}$ Finally, the results of the study showed that abrupt substitution yielded better results for chronic users of sedative-hypnotics. 
Others, however, do not share this view. Two reviews by Kripke ${ }^{54,55}$ stated that: "after tolerance develops, longterm use of sedative-hypnotics may make sleep worse"; "prescriptions for sedative-hypnotics should not be renewed for long-term use"; "chronic users of sedative-hypnotics should be gradually withdrawn, combined with possible provision of cognitive-behavioral therapy"; and, "cognitivebehavioral therapies are the best-supported treatment for chronic insomnia." The above recommendation is partly based on a paper published in 1998 on "Mortality hazard associated with prescription hypnotics" 54 but made no attempt to balance for the impact of sleep disruption per se or the reasons why people with long-term illness may require sedative-hypnotic treatment.

\section{Insomnia evaluation and diagnostic tools}

Polysomonography (PSG) is the accepted gold standard for sleep assessment. ${ }^{67}$ However, in light of the variable first night effect ${ }^{68}$ where one night in the sleep clinic may inaccurately reflect (either more positively or negatively) the usual sleep of insomnia patients, and the fact that most patients complaining of poor sleep quality do not show PSG abnormalities, ${ }^{69} \mathrm{PSG}$ is not recommended for the routine evaluation of chronic insomnia and is only indicated where there are signs or symptoms of another sleep disorder. ${ }^{70}$

Actigraphy is a practical alternative to PSG for objective assessment of sleep in insomnia patients, especially where there is a possible circadian rhythm disorder. ${ }^{71,72}$ As actigraphy is an in-home test it is much more economical than PSG and has the further advantage of recording sleep over weeks or months as opposed to one night of PSG, which will avoid first night effects and allow for the differential diagnosis of circadian rhythm disorders. ${ }^{71}$ Where objective sleep measurements are required to determine treatment outcome, clinical guidelines ${ }^{72}$ recommend the use of actigraphy to evaluate the treatment response in patients with insomnia or insomnia in combination with depression. ${ }^{72}$ However, more studies are needed to establish the clinical value, validity, and reliability of actigraphy in the management of insomnia.

Given the subjective nature of insomnia, questionnaires assessing severity of insomnia, ${ }^{73}$ sleep quality, ${ }^{74}$ or the non-restorative nature of sleep (we have developed and are currently validating a Non-Restorative Sleep scale) can provide useful additional clinical information. Fatigue, but not excessive daytime sleepiness, is often reported in those with insomnia and scales such as the Fatigue Adjective Checklist $(\text { FACES })^{75}$ or the Fatigue Severity Scale ${ }^{76}$ can be useful when determining response to treatment. Further, with minimal training, questionnaires can be easily and cheaply applied in primary care settings.

\section{Conclusion}

Chronic insomnia is a common complaint in primary care worldwide ${ }^{77}$ and insomnia has become increasingly recognized as a chronic disorder that requires treatment over the long-term. ${ }^{78}$ We think the case for long-term sedativehypnotic use is strong, if these drugs are used appropriately. In other branches of medicine objective data would be used, where available. One would not treat patients with tuberculosis for 6 months without chest X-rays or provide prescriptions for hypertension without measuring blood pressure. We believe that, in the case of long-term sedative-hypnotic use, thorough clinical assessment should be complemented by objective and subjective assessment. Unfortunately, as most patients with insomnia are managed in primary care, the sleep community needs to proactively involve general practitioners in sleep educational activities.

It is also perverse that many physicians have been persuaded that it is inappropriate to prescribe sedativehypnotics over the long-term and therefore prescribe other agents with sedative-hypnotic side effects (eg, anxiolytics and antidepressants). ${ }^{79}$ However, a similar deficit in knowledge exists regarding the long-term use of antidepressants, and even shorter-term use is not without serious side effects. ${ }^{80-83}$ We therefore think it is misguided to use a different class of drugs simply for the side effect of inducing sleep.

It is reasonable to prescribe sedative-hypnotics for a year at a time if one has the benefit of objective testing. Think of an optometrist's test or having a check-up at the dentist: any treatment in both cases depends on a thorough evaluation. Why should a sleep disorder with all its medical ramifications be held to lower standard? While some of these conclusions may be controversial they are not very far from "respected" and received wisdom. For example, the National Institute of Mental Health consensus development conference on drugs and insomnia addressed the issue of pharmacological therapy, to wit: "Unless high doses of benzodiazepines are used for several weeks, or low doses for a number of months, physical dependence is not likely to be of sufficient severity to cause major clinical problems."10

We should not let the politics of expedience or polemics of the media dictate what is best for patients. A serious attempt to address unanswered questions in the studies executed to date is warranted. We should avoid the phenomenon of faulty reasoning described at the beginning of this article. On a 
positive note, there has been a shift in the thinking about insomnia medication in the sleep community, perhaps given that the safety of insomnia medications has also improved. In 2005 the FDA approved three drugs and no restrictions were placed on their therapeutic timeline. The three drugs approved are eszopiclone, ramelteon, and zolpidem extended release. ${ }^{78}$ However, failure of physicians to effectively manage longterm insomnia in patients tend to promote use of over-thecounter medications, not only increasing the chance of drug interactions but also resulting in a greater medical risk and potentially producing more side effects than the prescribed sedative-hypnotics.

That more research is needed is to understate the issue. In a State of the Science Conference Statement on "Manifestations and Management of Chronic Insomnia in Adults" the National Institutes of Health ${ }^{84}$ proposed that there is a need for long-term efficacy studies of treatment for chronic insomnia and suggested including an assessment of quality of life, the impact on work performance, and other outcome measures in addition to quantitative sleep measurements. ${ }^{38}$ There are also limited studies for the treatment of insomnia in the elderly patients where maintenance insomnia is still a frequently reported problem. To date, ezopiclone is the only medication that has received FDA approval in 2004 for maintenance insomnia. ${ }^{21}$ The study by Walsh and colleagues ${ }^{38}$ showed that sedative-hypnotics can be used for long-term treatment of insomnia but research is still needed to provide evidence-based guidelines on how to treat patients with more complex medical and psychiatric issues. Such patients, while widespread in clinical practice, are routinely excluded from clinical trials. Clinically, the management of patients with chronic insomnia remains a challenge but one worth tackling with an open mind.

\section{Disclosure}

The authors report no conflicts of interest in this work.

\section{References}

1. Wake up America: a national sleep alert: report of the National Commission on Sleep Disorders Research. Washington, DC: Department of Health and Human Services; National Commission on Sleep Disorders Research (US); 1993.

2. Montplaisir J, Hawa R, Moller H, et al. Zopiclone and zaleplon vs benzodiazepines in the treatment of insomnia: Canadian consensus statement. Hum Psychopharmacol. 2003;18(1):29-38.

3. Mellinger GD, Balter MB, Uhlenhuth EH. Insomnia and its treatment. Prevalence and correlates Arch Gen Psychiatry. 1985;42(3):225-232.

4. Ford DE, Kamerow DB. Epidemiologic study of sleep disturbances and psychiatric disorders. An opportunity for prevention? JAMA. 1989;262(11):1479-1484

5. Manabe K, Matsui T, Yamaya M, et al. Sleep patterns and mortality among elderly patients in a geriatric hospital. Gerontology. 2000; 46(6):318-322.
6. Mallon L, Broman JE, Hetta J. Sleep complaints predict coronary artery disease mortality in males: a 12-year follow-up study of a middle-aged Swedish population. J Intern Med. 2002;251(3):207-216.

7. Leppavuori A, Pohjasvaara T, Vataja R, et al. Insomnia in ischemic stroke patients. Cerebrovas Dis. 2002;14(2):90-97.

8. Chilcott LA, Shapiro CM. The socioeconomic impact of insomnia An overview. Pharmacoeconomics. 1996;10 Suppl 1:1-14.

9. No authors listed. Insomnia: assessment and management in primary care. National heart, lung, and blood institute working group on insomnia. $\mathrm{Am}$ Fam Physician. 1999;59(11):3029-3038.

10. Consensus conference. Drugs and insomnia. The use of medications to promote sleep. JAMA. 1984;251(18):2410-2414.

11. Czeisler CA, Richardson GS. Detection and assessment of insomnia. Clin Ther. 1991;13(6):663-679.

12. Trajanovic N, Radivojevic V, Kaushansky Y, Shapiro CM. Positive sleep state misperception - a new concept of sleep misperception. Sleep Med. 2007;8(2):111-118.

13. Sivertsen B, Omvik S, Pallesen S, et al. Cognitive behavorial therapy versus zopiclone for the treatment of chronic primary insomnia in older patients. JAMA. 2006;295(24):2851-2858.

14. Lacks P, Morin C. Recent advances in the assessment and treatment of insomnia. J Consult Clin Psychol. 1992;6(4):586-594.

15. Morin C. Combined therapeutics for insomnia: should our first approach be behavioral or pharmacological? Sleep Med. 2006; 7(Suppl 1):S15-S19.

16. Morin AK. Strategies for treating chronic insomnia. Am J Manag Care. 2006;12(8 Suppl):S230-S245.

17. Edinger JD, Krystal AD. Sub typing primary insomnia: is sleep state misperception a distinct clinical entity? Sleep Med Rev. 2003; 7(3):203-214.

18. Riemann D. The hyperarousal model of insomnia: a review of the concept and its evidence. Sleep Med Rev. 2010;14(1):19-31.

19. Kennedy S, Parikh SV, Shapiro CM. Defeating Depression. Thornhill, ON: Joli Joco Publications, Inc.; 1998.

20. Ohayon MM, Shapiro CM, Kennedy SH. Differentiating DSM-IV anxiety and depressive disorders in the general population: comorbidity and treatment consequences. Can J Psychiatry. 2000;45(2):166-172.

21. Benca RM. Diagnosis and treatment of chronic insomnia: a review. Psychiatr Serv. 2005;56(3):332-343.

22. Fava M, McCall WV, Krystal A, et al. Eszopiclone co-administered with fluoxetine in patients with insomnia coexisting with major depressive disorder. Biol Psychiatry. 2006;59(11):1052-1060.

23. Shen J, Moller HJ, Wang X, et al. Mirtazapine a sedating antidepressant and improved driving safety in patients with major depressive disorder: a prospective randomized control trial of 28 patients. J Clin Psychiatry. 2009;70(3):370-377.

24. Levitan RD, Shen JH, Jinda RL, Driver HS, Kennedy SH, Shapiro CM. Preliminary randomized double-blind placebo-controlled trial of tryptophan combined with fluoxetine to treat major depressive disorder: antidepressant and hypnotic effects. $J$ Psychiatry Neurosci. 2000;25(4):337-346.

25. Anderson IM, Ferrier IN, Baldwin RC. Evidence-based guidelines for treating depressive disorders with antidepressants: a revision of the 2000 British Association for Psychopharmacology guidelines. J Psychopharmacol. 2008;22(4):343-396.

26. Benca RM. Consequences of insomnia and its therapies. $J$ Clin Psychiatry. 2001;62 Suppl 10:33-38.

27. Kramer M. Hypnotic medication in the treatment of chronic insomnia: non nocere! Doesn't anyone care? Sleep Med Rev. 2000; 4(6):529-541.

28. McAndrews MP, Kayumov L, Phillipson R, Shapiro CM. Self-report of memory and affective dysfunction in association with medication use in a sample of individuals with chronic sleep disturbance. Hum Psychopharmacol. 2000;15(8):583-587.

29. McAndrews MP, Weiss RT, Sandor P, Taylor A, Carlen PL, Shapiro CM. Cognitive effects of long-term benzodiazepine use in older adults. Hum Psychopharmacology. 2003;18(1):51-57. 
30. Lucki I, Rickels K, Geller AM. Chronic use of benzodiazepines and psychomotor and cognitive test performance. Psychopharmacology (Berl). 1986;88(4):426-433.

31. Golombok S, Moodley P, Lader M. Cognitive impairment in long-term benzodiazepine users. Psychol Med. 1988;18(2):365-374.

32. Hanlon JT, Horner RD, Schmader KE, et al. Benzodiazepine use and cognitive function among community-dwelling elderly. Clin Pharmacol Ther. 1998;64(6):684-692.

33. Adam K, Adamson L, Brezinova V, HunterWM. Nitrazepam: lastingly effective but trouble on withdrawal. Br Med J. 1976;1(6025):1558-1560.

34. Oswald I, French C, Adam K, Gilham J. Benzodiazepine hypnotics remain effective for 24 weeks. Br Med J (Clin Res Ed). 1982;284(6319): $860-863$.

35. Scharf MB, Roth T, Vogel GW, et al. A multicenter, placebo-controlled study evaluating zolpidem in the treatment of chronic insomnia. J Clin Psychiatry. 1994;55(5):192-199.

36. Elie R, Ruther E, Farr I, Emilien G, Salinas E. Sleep latency is shortened during 4 weeks of treatment with zaleplon, a novel non benzodiazepine hypnotic. Zaleplon Clinical Study Group. J Clin Psychiatry. 1999;60(8):536-544.

37. Allen RP, Mendels J, Nevins DB, et al. Efficacy without tolerance or rebound insomnia for midazolam and temazepam after use for one to three months. J Clin Pharmacol. 1987;27(10):768-775.

38. Walsh JK, Krystal AD, Amato DA, et al. Nightly treatment of primary insomnia with eszopiclone for six months: effect on sleep, quality of life, and work limitations. Sleep. 2007;30(8):959-968.

39. Roth T, Walsh JK, Krystal A, Wessel T, Roehrs TA. An evaluation of the efficacy and safety of eszopiclone over 12 months in patients with chronic primary insomnia. Sleep Med. 2005;6(6):487-495.

40. Pakes GE, Brogden RN, Heel RC, Speight TM, Avery GS. Triazolam: a review of its pharmacological properties and therapeutic efficacy in patients with insomnia. Drugs. 1981;22(2):81-110.

41. Maarek L, Cramer P, Attali P, Coquelin JP, Morselli PL. The safety and efficacy of zolpidem in insomniac patients: a long-term open study in general practice. $J$ Int Med Res. 1992;20(2):162-170.

42. Schenck CH, Mahowald MW. Long-term, nightly benzodiazepine treatment of injurious parasomnias and other disorders of disrupted nocturnal sleep in 170 adults. Am J Med. 1996;100(3): 333-337.

43. Ancoli-Israel S, Richardson GS, Mangano RM, Jenkins L, Hall P, Jones WS. Long-term use of sedative hypnotics in older patients with insomnia. Sleep Med. 2005;6(2):107-113.

44. Krystal AD, Walsh JK, Laska E, et al. Sustained efficacy of eszopiclone over 6 months of nightly treatment: results of a randomized, double-blind, placebo-controlled study in adults with chronic insomnia. Sleep. 2003;26(7):793-799.

45. Jacobs GD. Is eszopiclone appropriate and effective for the long-term clinical management of insomnia. Sleep. 2004;27(2):345.

46. Morin CM, Bastien CH, Brink D, Brown TR. Adverse effects of temazepam in older adults with chronic insomnia. Hum Psychopharmacol. 2003;18(1):75-82.

47. Regestein QR, Reich P. A sleep clinic within a general hospital psychiatry service. Gen Hosp Psychiatry. 1980;2(2):112-117.

48. Gillin JC, Byerley WF. Drug therapy: the diagnosis and management of insomnia. N Engl J Med. 1990;322(4):239-248.

49. Terzano MG, Rossi M, Palomba V, Smerieri A, Parrino L. New drugs for insomnia: comparative tolerability of zopiclone, zolpidem and zaleplon. Drugs. 2003;26(4):261-282.

50. Shah R, Uren Z, Baker A, Majeed A. Deaths from antidepressants in England and Wales 1993-1997: analysis of a new national database. Psychol Med. 2001;31(7):1203-1210.

51. Bolster M, Curran J, Busuttil A. A five year review of fatal self-ingested overdoses involving amitriptyline in Edinburgh 1983-1987. Hum Exp Toxicol. 1994;13(1):29-31.

52. Henry JA, Alexander CA, Sener EK. Relative mortality from overdose of antidepressants. BMJ. 1995;310(6974):221-224.
53. Shapiro CM, Parry MR. Is unemployment a cause of Parasuicide. Br Med J (Clin Res Ed). 1984;289(6458):1622.

54. Kripke DF, Klauber MR, Wingard DL, Fell RL, Assmus JD, Garfinkel L. Mortality hazard associated with prescription hypnotics. Biol Psychiatry. 1998;43(9):687-693.

55. Kripke DF. Chronic hypnotic use: deadly risks, doubtful benefit. Sleep Med Rev. 2000;4(1):5-20.

56. Bliwise DL. Treating insomnia: pharmacological and tnonpharmacological approaches. J Psychoactive Drugs. 1991;23(4):335-341.

57. Shapiro CM, MacFarlane JG, MacLean AW. Alleviating sleep-related discontinuance symptoms associated with benzodiazepine withdrawal: a new approach. J Psychosom Res. 1993;37 Suppl 1:55-57.

58. Shapiro CM, Ohayon MM, Ebrahim I, Grunstein R. Fighting Fatigue and Sleepiness. Thornhill, ON: Joli Joco Publications, Inc.; 2005.

59. Hajak G, Geisler P. Experience with zolpidem 'as needed' in primary care settings. CNS Drugs. 2004;18 Suppl 1:35-40.

60. Vallieres A, Morin CM, Guay B, Bastien CH, LeBlanc M. Sequential treatment for chronic insomnia: a pilot study. Behav Sleep Med. 2004;2(2):94-112.

61. Morin CM. Cognitive-behavioral approaches to the treatment of insomnia. J Clin Psychiatry. 2004;65 Suppl 16:33-40.

62. Morin CM, Belanger L, Bastien C, Vallieres A. Long-term outcome after discontinuation of benzodiazepines for insomnia: a survival analysis of relapse. Behav Res Ther. 2005;43(1):1-14.

63. Morin CM, Vallieres, A, Guay B, et al. Cognitive behavioral therapy, singly and combined with medication, for persistent insomnia. A randomized controlled trial. JAMA. 2009;301(19):2005-2015.

64. Shapiro CM, Sherman D, Peck DF. Withdrawal from benzodiazepines by initially switching to zopiclone. Eur Psychiatry. 1995;10(Suppl 3):145S-151S.

65. Shapiro C. Zopiclone as a catalyst to hypnotic withdrawal. In: Joyce D, Partinen M, Roth T, Ruther E, (Chairmen). Zopiclone: From Clinical Practice to Quality of Life. Copenhagen, Denmark: AEP; 1994:31.

66. Lemoine P, Ohayon MM. Is hypnotic withdrawal facilitated by the transitory use of a substitute drug? Prog Neuropsychopharmacol Biol Psychiatry. 1997;21(1):111-124.

67. Kushida CA, Littner MR, Morgenthaler T, et al. Practice parameters for the indications for polysomnography and related procedures: an update for 2005. Sleep. 2005;28(4):499-521.

68. Hauri PJ, Olmstead EM. Reverse first night effect in insomnia. Sleep. 1989;12(2):97-105.

69. Backhaus J, Junghanns K, Broocks A, Riemann D, Hohagen F. Testretest reliability and validity of the Pittsburgh Sleep Quality Index in primary insomnia. J Psychosom Res. 2002;53(3):737-740.

70. Chesson A Jr, Hartse K, Anderson WM, et al. Practice parameters for the evaluation of chronic insomnia. An American Academy of Sleep Medicine report. Standards of Practice Committee of the American Academy of Sleep Medicine. Sleep. 2000;23(2):237-241.

71. Ancoli-Israel S, Cole R, Alessi C, Chambers M, Moorcroft W, Pollak CP. The role of actigraphy in the study of sleep and circadian rhythms. Sleep. 2003;26(3):342-392.

72. Morgenthaler T, Alessi C, Friedman L, et al. Practice parameters for the use of actigraphy in the assessment of sleep and sleep disorders: an update for 2007. Sleep. 2007;30(4):519-529.

73. Soldatos CR, Dikeos DG, Paparrigopoulos TJ. The diagnostic validity of the Athens Insomnia Scale. J Psychosom Res. 2003;55(3):263-267.

74. Buysse DJ, Reynolds CF 3rd, Monk TH, Berman SR, Kupfer DJ. The Pittsburgh Sleep Quality Index: a new instrument for psychiatric practice and research. Psychiatry Res. 1989;28(2):193-213.

75. Shapiro CM, Flanigan M, Fleming JA, et al. Development of an adjective checklist to measure five FACES of fatigue and sleepiness. Data from a national survey of insomniacs. J Psychosom Res. 2002;52(6): $467-473$.

76. Krupp LB, LaRocca NG, Muir-Nash J, Steinberg AD. The fatigue severity scale. Application to patients with multiple sclerosis and systemic lupus erythematosus. Arch Neurol. 1989;46(10):1121-1123. 
77. Léger D, Partinen M, Hirshkowitz M, Chokroverty S, Hedner J; EQUINOX (Evaluation of daytime QUality Impairment by Nocturnal awakenings in Outpatient's eXperience) Survey Investigators. Characteristics of insomnia in a primary care setting: EQUINOX survey of 5293 insomniacs from 10 countries. Sleep Med. 2010;11(10): 987-998.

78. Lieberman JA. Update on the safety considerations in the management of insomnia with hypnotics: incorporating modified release formulations into primary care. J Clin Psychiatry. 2007;9(1):25-31.

79. Roehrs T, Roth T. 'Hypnotic' prescription patterns in a large managed-care population. Sleep Med. 2004;5(5):463-466.

80. Virk S, Schwartz TL, Jindal S, Nihalani N, Jones N. Psychiatric medication induced obesity: an aetiologic review. Obes Rev. 2004; 5(3):167-170.
81. Fabian TJ, Amico JA, Kroboth PD, et al. Paroxetine-induced hyponatremia in older adults: a 12-week prospective study. Arch Intern Med. 2004;164(3):327-332.

82. Biswas PN, Wilton LV, Shakir SA. The pharmacovigilance of mirtazapine: results of a prescription event monitoring study on 13554 patients in England. J Psychopharmacol. 2003;17(1):121-126.

83. Luby ED, Singareddy RK. Long-term therapy with lithium in a private practice clinic: a naturalistic study. Bipolar Disord. 2003;5(1): 62-68.

84. National Institutes of Health. National Institute of Health Sate of the Science Conference statement on Manifestations and Management of Chronic Insomnia in Adults, June 13-15, 2005. Sleep. 2005;28(9):1049-1057.

\section{Publish your work in this journal}

Nature and Science of Sleep is an international, peer-reviewed, open access journal covering all aspects of sleep science and sleep medicine, including the neurophysiology and functions of sleep, the genetics of sleep, sleep and society, biological rhythms, dreaming, sleep disorders and therapy, and strategies to optimize healthy sleep. The journal welcomes

\section{Dovepress}

original research, clinical \& epidemiological studies, reviews \& evaluations, case reports and extended reports. The manuscript management system is completely online and includes a very quick and fair peerreview system, which is all easy to use. Visit http://www.dovepress.com/ testimonials.php to read real quotes from published authors. 